\title{
Corrigendum
}

\section{The emerging role of RNAs in DNA damage repair}

\author{
Ben R Hawley, Wei-Ting Lu, Ania Wilczynska and Martin Bushell
}

Cell Death and Differentiation (2017) 24, 1989; doi:10.1038/cdd.2017.146; published online 1 September 2017

Correction to: Cell Death and Differentiation (2017) 24, 580-587; doi:10.1038/cdd.2017.16; published online 24 February 2017

Since the publication of this paper, the authors have noticed a small number of citation errors and a couple of phrases that may be open to misinterpretation.

The authors would like to apologize for any inconvenience caused.

- Page 582: 'Moreover, the participation of key downstream effectors in the RNAi pathway, namely the Argonautes, is also contested. 8,10,11,21, Remove citation 21 .

- Page 585: 'Cell lines carrying a DR-GFP integrated reporter (Figure 4) were incubated with a pre-extracted small RNA fraction from damaged or undamaged cells. ${ }^{9,11}$. Citations should be changed to 8 and 10 .
- Page 585: 'Depletion of Drosha or Dicer in cells carrying this transgene following 2 days of damage resulted in a reduction in GFP-positive cells indicating that $\mathrm{HR}$ was impaired. 9,10, Also add reference 8.

- Page 585: 'Nevertheless, it is important to remember that this method was not the only approach used by the authors.' We wish to end the sentence earlier to read 'Nevertheless, it is important to remember that this method was not the only approach used.'

- Table 1, second row, the remarks section 'required for proficient repair of DNA damage...' should instead be 'required for proficient signalling and repair of DNA damage...'.

- Page 586: 'However, the small RNA produced in mammalian cells, termed diRNAs, are reported to be directly involved in the repair process...' should instead be 'However, the small RNA produced in mammalian cells, termed diRNAs, are reported to be directly involved in the signalling and repair process...' 